\title{
Biolog for the determination of diversity in microbial communities
}

\author{
J van Heerden*, C Korf, MM Ehlers and TE Cloete \\ Department of Microbiology and Plant Pathology, Faculty of Biological and Agricultural Sciences, University of Pretoria, \\ Pretoria 0002, South Africa
}

\begin{abstract}
Diversity and dynamics of microbial communities have been analysed by culture-dependent methods, which exclude the majority of fastidious microbes due to the selective nature of the media. Molecular methods have been used to determine diversity of microbial communities, but indicate the genetic complexity within a community. An alternative approach is to examine components of functional biodiversity (i.e. substrate utilisation), for which there exists a reasonable chance of detecting patterns, which could be related to the functional diversity of the species present in the community. In this study, different carbon source profiles were generated by inoculating Biolog GN and GP microtitre plates, with different dilutions of microbial communities. The high number of substrates utilised at the lower dilutions $\left(10^{-1}\right.$ and $\left.10^{-2}\right)$ indicated a high functional diversity in the communities tested. This, however, did not necessarily reflect the evenness of the functionality. Functional evenness of each species was reflected upon further dilution. Our results indicated differences in the functional diversity of the microbial communities amongst some of the natural environments studied. The results indicated that evenness and dominance can be demonstrated by mixtures of cultures as well as in natural environments.
\end{abstract}

\section{Introduction}

The majority of micro-organisms in their natural habitat cannot be cultured and remain unidentified (Cloete et al., 1992; Haldeman and Amy, 1993; Wagner et al., 1993). This has led to a lack of knowledge of microbial community composition and function. Recently, molecular techniques have been used in microbial ecology studies in an attempt to overcome the limitations of culture techniques (Pace et al., 1986; Wagner et al., 1993; Amann et al., 1995; Muyima et al., 1997). These techniques require a high level of expertise and sophistication and are often tedious (Schwieger and Tebbe, 1998). The techniques are qualitative and not quantitative (Muyima et al., 1997). Hence, there is a need for techniques that characterise microbial communities without the reliance on selective culturing and which are less complex than molecular techniques.

Microbial communities can be considered to be systems containing information (data), which is related primarily to microbial diversity. The higher the diversity, the more the information. By employing molecular techniques, it is currently attempted to translate this information into meaningful data. Molecular techniques rely on information in a microbial community which are extracted as molecules from the genome of the different members of the community (Torsvik et al., 1990). This would indicate genetic complexity, which could, in turn, be attributed to microbial diversity in the system. A more simplistic and effective method is, however, required to achieve this translation and interpretation of data.

In any given system one or more species exists, each performing a certain function. The more species, the more functions related to their metabolism. It can be said that a specific microbial community has a specific metabolic capacity. Functional diversity can be determined in terms of the presence, absence or rate of substrate utilisation (Griffiths et al., 1997). Griffiths et al. (1997) indicated

\footnotetext{
* To whom all correspondence should be addressed.

푱(012) 319-2534; fax: (012) 325-5550;

e-mail: jvanheer@medic.up.ac.za

Received 27 September 2000; accepted in revised form 13 September 2001.
}

that there could be changes in microbial community structure with no changes in function, but that function was affected below a certain level of species diversity (microbial capacity). One of the objectives of this study was to determine the microbial capacity of the community to utilise certain selected substrates to functional diversity. The hypothesis is that the more substrates utilised, the higher the diversity, due to the collective action of individual species.

Any one organism will not necessarily utilise all the available substrates in a system, nor does the utilisation of some of the substrates suggest that this is the complete set of substrates which a particular organism can use because of:

- competition, which might suppress the activity of a particular organism;

- dominance where one organism utilises all the substrates in such a way that the contribution of other organisms to substrate utilisation is overshadowed and goes unnoticed;

- substrates which might not match the metabolic activity of a particular organism (meaning that it will not show up on the analysis);

- the system that might be selective, i.e. it would only allow the metabolic activities of aerobic or facultatively anaerobic, heterotrophic and copiotrophic micro-organisms which are capable of growing at sufficient rates on the substrates;

- dependence on the abundance of each species, i.e. the organisms present in higher numbers will be able to utilise the carbon sources easier than organisms present in low numbers;

- inoculum density which has an influence on the tempo and occurrence of colour development due to the growth rates of organisms on different substrates;

- incubation time that influences substrate utilisation of a community due to individual growth rates of the microorganisms on different substrates (Wünsche et al., 1995);

- antagonistic interactions between organisms, where one organism inhibits another organism's growth and therefore its 
ability to utilise the carbon sources; and

- toxic effects of the redox dyes may inhibit the growth of the organism (Ullrich et al., 1996).

The purpose of this study was not to detect each and every metabolic reaction of all the individuals in the community, but rather to determine the collective pattern for a specific community (Van Heerden et al., 2001). The metabolic pattern will indicate one of three possible scenarios:

- a high species diversity which should result from a higher relative number of substrates being utilised;

- evenness which would result in the same number of substrates utilised upon dilution; and

- upon dilution, some organisms will be lost from the community, causing a decrease in species diversity (Van Heerden et al., 2001). The extent of the reduction of the possibilities upon dilution, should theoretically indicate the extent of the community structure (Van Heerden et al., 2001). The key lies in the interpretation of the results.

One possible system that affords the opportunity to generate metabolic patterns for a specific community is the Biolog system (Garland and Mills, 1991; Haack et al., 1994; Winding, 1994; Zak et al., 1994; Bossio and Scow, 1995; Ellis et al., 1995; Wünsche et al., 1995; Guckert et al., 1996; Insam et al., 1996; Smalla et al., 1996; Garland, 1997; Glimm et al., 1997; Heuer and Smalla, 1997; Kersters et al., 1997; Engelen et al., 1998). This system relies on the potential utilisation of 95 substrates in a microwell plate by the community (Biolog, Inc., Hayward, CA). Unlike traditional culture - dependent methods, which are generally selective for the component of the microbial community that has to be cultured, the Biolog method can reflect the activities of a broad range of bacteria (Zak et al., 1994). Therefore, the Biolog system is considered to be a battery of metabolic tests to use for generating a database for a specific community as discussed. The aim of this study was to inoculate Biolog microtitre plates with samples from natural and artificially-created microbial communities and to interpret the microbial community level carbon source utilisation patterns and to relate this to functional diversity and evenness (distribution of species abundance within the community).

\section{Experimental}

\section{Biolog microtitre plates}

Biolog GN and GP microtitre plates (Biolog Inc., Hayward, CA) were used in this study.

\section{Bacterial strains and inoculum preparation}

Studies were performed using mixtures of the following pure cultures: Serratia marcescens (DSM 30121), Staphylococcus aureus (ATCC 196E), Pasteurella multocida (LMG 2851), Alcaligenes eutrophus (DSM 531), Acinetobacter calcoaceticus (DSM 588), Rhodococcus equi (LMG 5358) and Micrococcus luteus (DSM 20030). The LMG culture was obtained from the LMG culture collection (Gent, Belgium), DSM cultures from Germany and the ATCC culture from the USA. Serratiamarcescens was used as a pure culture suspension (using $10^{-5}$ to $10^{-8}$ dilutions) in order to simulate dominance, as one might expect in an extreme habitat. Profiles of diversity, presenting evenness (ME) and unevenness (MU) were obtained by inoculating GN Biolog microtitre plates with different mixtures of pure cultures over a range of dilutions (Table 1 and 2 ).

\section{Plate counts}

Viable counts were determined by the spread plate procedure. Samples were serially diluted in $0.85 \%$ (w/v) $\mathrm{NaCl}$ (up to $10^{-4}$ dilution) and $100 \mu$ l of each dilution was plated on appropriate media. Pasteurella multocida was enumerated on brain heart infusion agar (BHI, Merck) and the other cultures on nutrient agar (Merck). The plates were incubated at $28^{\circ} \mathrm{C}$ and colonies were counted after 24 h (S.marcescens, P.multocida, A.eutrophus, A. calcoaceticus) and $48 \mathrm{~h}$ (S.aureus, R.equi and M.luteus). Hot water spring and activated sludge water samples were cultured on $\mathrm{R}_{2} \mathrm{~A}$ agar (Merck) and incubated at $37^{\circ} \mathrm{C}$ and colonies were counted after 24 and $48 \mathrm{~h}$.

\section{Sample sources and collection}

Mixed liquor grab samples $(1 \ell)$ were drawn from the anaerobic zones of the following activated sludge plants operated by: Heidelberg (HDLB), Vlakplaats (VLKP), Tsakane (TSKN) and Hartebeesfontein (HBFT), Gauteng, South Africa (ERWAT). Hot water spring samples were collected from Warmbaths (South Africa). All samples were collected in sterile screw-capped plastic bottles and samples were analysed within $12 \mathrm{~h}$ after collection.

\section{Inoculation and incubation of Biolog microtitre plates}

\section{Pure cultures}

Pure culture inocula for microplates were obtained by growing pure cultures on nutrient agar with the exception of P.multocida, which was grown on BHI agar. S.marcescens, P.multocida, A.eutrophus and A. calcoaceticus were scraped from plates after $24 \mathrm{~h}$ and S.aureus, R.equi and M.luteus after $48 \mathrm{~h}$ growth. Pure cultures were mixed and suspended in sterile saline $(0.85 \%(\mathrm{w} / \mathrm{v})$ $\mathrm{NaCl})$. These mixtures were serially diluted $\left(10^{-1}\right.$ to $10^{-4}$ dilution $)$ before inoculation into Biolog microtitre plates. Biolog GN microtitre plate wells were inoculated with $150 \mu \ell$ of the cell suspensions (dilutions of the bacterial cultures) (Engelen et al., 1998). Microtitre plates were incubated aerobically in the dark at $28^{\circ} \mathrm{C}$ without agitation. Colour formation in the individual cells of the microtitre plates was measured at 405 and $590 \mathrm{~nm}$ with an Anthos reader 2001 (Anthos Labtec Instruments). Duplicate readings of the microtitre plates were made after $24 \mathrm{~h}, 48 \mathrm{~h}$ and $72 \mathrm{~h}$ of incubation. Plates were also studied visually.

\section{Hot water spring}

Samples obtained from a hot water spring were diluted with sterile saline $(0.85 \%(\mathrm{w} / \mathrm{v}) \mathrm{NaCl})$. These dilutions were used as inoculum for Biolog GN microtitre plates. Biolog GN microtitre plates were inoculated with $150 \mu$ o of the cell suspensions (Kersters et al., 1997). Microtitre plates were incubated aerobically in the dark at $37^{\circ} \mathrm{C}$ without agitation. Colour formation in the individual cells of the microtitre plates was measured at $590 \mathrm{~nm}$ with an Anthos reader 2001 (Anthos Labtec Instruments). Duplicate readings of the microtitre plates were made after $24 \mathrm{~h}, 48 \mathrm{~h}$ and $72 \mathrm{~h}$ of incubation.

\section{Activated sludge}

Biolog GN and GP microtitre plates were inoculated with activated sludge obtained from activated sludge systems in the Gauteng region, South Africa. The activated sludge suspensions were diluted $\left(10^{-1}\right.$ to $10^{-4}$ dilution) with sterile saline $(0.85 \%(\mathrm{w} / \mathrm{v}) \mathrm{NaCl})$ and 


\begin{tabular}{|c|c|c|c|c|c|c|}
\hline \multicolumn{7}{|c|}{$\begin{array}{l}\text { TABLE } 1 \\
\text { Mixtures of bacterial cultures used to determine the effect of evenness in biolog } \\
\text { microtitre plates }\end{array}$} \\
\hline $\begin{array}{l}\text { Mixtures } \\
\text { of bacterial } \\
\text { cultures* }^{*}\end{array}$ & $\begin{array}{l}\text { S. aureus } \\
\left(\mathrm{cfu} \cdot \mathrm{m} \ell^{-1}\right)^{\#}\end{array}$ & $\begin{array}{l}\text { P. multocida } \\
\left(\mathrm{cfu} \cdot \mathrm{m} \ell^{-1}\right)^{\#}\end{array}$ & $\begin{array}{l}\text { A. eutrophus } \\
\left(\mathrm{cfu} \cdot \mathrm{m} \ell^{-1}\right)^{\#}\end{array}$ & $\begin{array}{l}\text { A. calco- } \\
\text { aceticus } \\
\left(\mathrm{cfu} \cdot \mathrm{m}^{-1}\right)^{\#}\end{array}$ & $\begin{array}{l}\text { M. luteus } \\
\left.\text { (cfu-m } \ell^{-1}\right)^{\#}\end{array}$ & $\begin{array}{c}\text { R. equi } \\
\left(\mathrm{cfu} \cdot \mathrm{m}^{-1}\right)^{\#}\end{array}$ \\
\hline $\begin{array}{l}\text { ME0 } \\
\text { ME-1 } \\
\text { ME-2 } \\
\text { ME-3 }\end{array}$ & $\begin{array}{l}10^{7} \\
10^{6} \\
10^{5} \\
10^{4}\end{array}$ & $\begin{array}{l}10^{7} \\
10^{6} \\
10^{5} \\
10^{4}\end{array}$ & $\begin{array}{l}10^{7} \\
10^{6} \\
10^{5} \\
10^{4}\end{array}$ & $\begin{array}{l}10^{7} \\
10^{6} \\
10^{5} \\
10^{4}\end{array}$ & $\begin{array}{l}10^{7} \\
10^{6} \\
10^{5} \\
10^{4}\end{array}$ & $\begin{array}{l}10^{7} \\
10^{6} \\
10^{5} \\
10^{4}\end{array}$ \\
\hline \multicolumn{7}{|c|}{$\begin{array}{l}\text { * Mixtures of bacterial cultures representing evenness (ME) used as inoculum for GN Biolog } \\
\text { microtitre plates. } \\
\text { \# Resuspended colonies were used to determine cfu } \cdot \mathrm{m}^{-1} \text { and estimated numbers of bacteria } \\
\text { used in Biolog microtitre plates. }\end{array}$} \\
\hline
\end{tabular}

\begin{tabular}{|c|c|c|c|c|c|c|}
\hline \multicolumn{7}{|c|}{$\begin{array}{l}\text { TABLE } 2 \\
\begin{array}{l}\text { Mixtures of bacterial cultures used to determine the effect of unevenly distributed } \\
\text { micro-organisms in biolog microtitre plates }\end{array}\end{array}$} \\
\hline $\begin{array}{l}\text { Mixtures } \\
\text { of bacterial } \\
\text { cultures* }\end{array}$ & $\begin{array}{l}\text { S. aureus } \\
\left(\mathrm{cfu} \cdot \mathrm{m} \ell^{-1}\right)^{\#}\end{array}$ & $\underset{\left(\text { Pfu } \cdot \mathrm{m}^{-1}\right)^{\#}}{\text { P. multocida }}$ & $\begin{array}{l}\text { A. eutrophus } \\
\left(\mathrm{cfu} \cdot \mathrm{m} \ell^{-1}\right)^{\#}\end{array}$ & $\begin{array}{l}\text { A. calco- } \\
\text { aceticus } \\
\left(\text { cfu-m } \ell^{-1}\right)^{\#}\end{array}$ & $\begin{array}{l}\text { M. luteus } \\
\left(\text { cfu-m } \ell^{-1}\right)^{\#}\end{array}$ & $\begin{array}{c}\text { R. equi } \\
\left(\mathrm{cfu} \cdot \mathrm{m} \ell^{-1}\right)^{\#}\end{array}$ \\
\hline $\begin{array}{l}\text { MU0 } \\
\text { MU-1 } \\
\text { MU-2 } \\
\text { MU-3 }\end{array}$ & $\begin{array}{l}10^{5} \\
10^{4} \\
10^{3} \\
10^{2}\end{array}$ & $\begin{array}{l}10^{4} \\
10^{3} \\
10^{2} \\
10^{1}\end{array}$ & $\begin{array}{l}10^{5} \\
10^{4} \\
10^{3} \\
10^{2}\end{array}$ & $\begin{array}{l}10^{6} \\
10^{5} \\
10^{4} \\
10^{3}\end{array}$ & $\begin{array}{l}10^{4} \\
10^{3} \\
10^{2} \\
10^{1}\end{array}$ & $\begin{array}{l}10^{5} \\
10^{4} \\
10^{3} \\
10^{2}\end{array}$ \\
\hline \multicolumn{7}{|c|}{$\begin{array}{l}\text { * Mixtures of pure bacterial cultures representing unevenness (MU) used as inoculum for GN } \\
\text { Biolog microtitre plates. } \\
\text { Resuspended colonies were used to determine cfu } \cdot \mathrm{m}^{-1} \text { and estimated number of bacteria } \\
\text { used in Biolog microtitre plates. }\end{array}$} \\
\hline
\end{tabular}

used as inoculum for Biolog GN and GP microtitre plates. Biolog GN and GP microtitre plate wells were inoculated with $150 \mu \ell$ of the activated sludge from each dilution (Zak et al., 1994). Microtitre plates were incubated aerobically (since strict anaerobic bacteria are not expected in activated sludge systems) in the dark at $21^{\circ} \mathrm{C}$ without agitation. Colour formation in the individual cells of the microtitre plates was measured with an Anthos reader 2001 (Anthos Labtec Instruments) at $590 \mathrm{~nm}$. Duplicate readings of the microtitre plates were made after $24 \mathrm{~h}, 48 \mathrm{~h}$ and $72 \mathrm{~h}$ of incubation and microtitre plates were also studied visually.

\section{Data handling}

Carbon source utilisation patterns were obtained by determining the percentage carbon sources utilised after a $72 \mathrm{~h}$ incubation period. Data were collected and collated using the Bionum Version 1.1 for Windows software (Applied Maths, Kortrijk, Belgium), as previously described (Van Heerden et al., 2001). The similarity between data sets was calculated by determining the simple matching coefficient $\left(\mathrm{S}_{\mathrm{sm}}\right)$ using the Bionum Version 1.1 for Windows software (Applied Maths, Kortrijk, Belgium). Analyses and dendrogram constructions were done using GelCompar (4.0) software(Applied Maths, Kortrijk, Belgium). The program clustered the samples using the unweighted pair group method of arithmetic averages (UPGMA).

\section{Results and discussion}

Colour development in each well of the GN and GP Biolog microtitre plates reflected the ability of the bacterial community to utilise that specific carbon source. According to Insam et al. (1996) $10^{4} \mathrm{cfu} \cdot \mathrm{m}^{-1}$ were required as an initial inoculum density for Biolog microtitre plate wells. Our results indicated that incubation periods of $24 \mathrm{~h}$ and $48 \mathrm{~h}$ were not sufficient for complete colour development in the Biolog microtitre plate wells with an initial inoculum density of $c a .10^{4} \mathrm{cfu} \cdot \mathrm{m}^{-1}$. The results indicated complete colour development after $72 \mathrm{~h}$ of incubation. All our results were based on a $72 \mathrm{~h}$ incubation period. A minimum initial inoculum density of $10^{4}$ $\mathrm{cfu} \cdot \mathrm{m}^{-1}$ was used in this study in compliance with the recommendations of Biolog. An absorbance reading of $590 \mathrm{~nm}$ is more effective than $405 \mathrm{~nm}$ absorbance to interpret Biolog microtitre plate results.

In this study we set out to indicate that metabolic diversity (substrate utilisation), as determined with the Biolog system, could be used to learn more about functional diversity (number of different substrates utilised) and evenness (distribution of species abundance within the community) in natural habitats. According to the manufacturer, the following percentage carbon sources are utilised in Biolog GN microtitre plates by the different bacterial strains used in this study: Serratia marcescens $60 \%$; Staphylococcus 


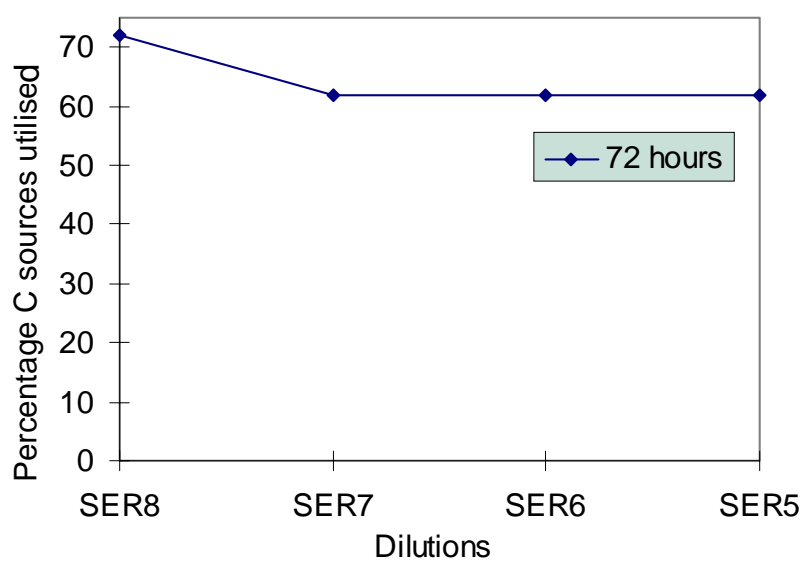

Figure 1(a)

Carbon source utilisation by Serratia marcescens over a range of dilutions $10^{8} \mathrm{cfu} \cdot \mathrm{m}^{1}$ (SER8), $10^{7} \mathrm{cfu} \cdot \mathrm{m} \ell^{1}$ (SER7), $10^{6} \mathrm{cfu} \cdot \mathrm{m} \ell^{1}$ (SER6) and $10^{5} \mathrm{cfu} \cdot \mathrm{m} \ell^{1}$ (SER5), representative of dominance.

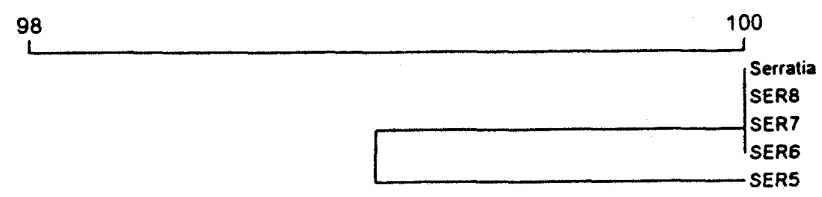

Figure 1(b)

Dendrogram of carbon source utilisation by Serratia marcescens over a range of dilutions $10^{8} \mathrm{cfu} \cdot \mathrm{m}^{11}$ (SER8), $10^{7} \mathrm{cfu} \cdot \mathrm{m} \ell^{1}$ (SER7), $10^{6} \mathrm{cfu} \cdot \mathrm{m} \ell^{1}$ (SER6) and $10^{5} \mathrm{cfu} \cdot \mathrm{m} \ell^{1}$ (SER5).

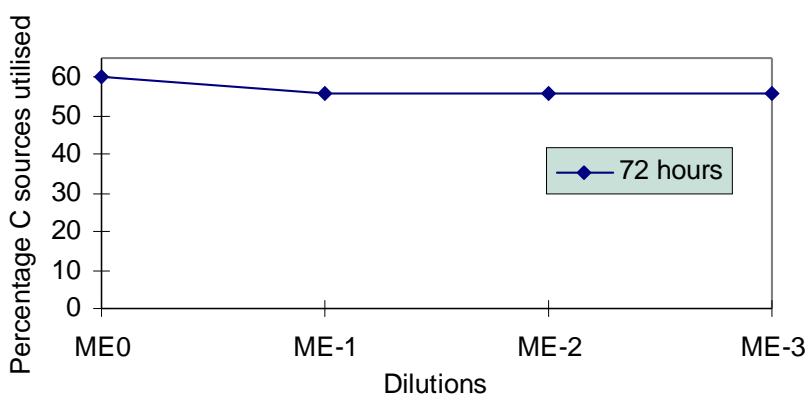

Figure 2(a)

Carbon source utilisation by the mixture of bacterial cultures (MEO, ME-1, ME-2 and ME-3), representative of evenness (TABLE 1). aureus 17\%; Pasteurella multocida 25\%; Alcaligenes eutrophus 35\%; Acinetobacter calcoaceticus 31\%; Micrococcus luteus 5\% and Rhodococcus equi $4 \%$.

The first artificial community contained only $S$. marcescens in order to simulate dominance, as one might expect in extreme environments (such as hot water springs, salt lakes and ice seas). Dilution of the S.marcescens culture did not result in a decline ( $<10 \%$ difference) in the number of substrates utilised (Fig 1a). This indicated, as postulated, that the presence of one organism (dominance) in a community would result in the same number of carbon sources utilised upon dilution, given that the initial inoculum density and incubation time were sufficient. This was substantiated by the dendrogram, which was constructed from these results (Fig 1b). A similarity of more than $98 \%$ was observed amongst these dilutions.

The second artificial community was constructed to reflect evenly (evenness) distributed species (Table 1). Upon dilution, a slight decline $(<10 \%$ difference) in the number of substrates utilised by the evenly distributed mixtures of bacterial cultures (Table 1) was observed after $72 \mathrm{~h}$ incubation (Fig. 2a). This indicated, that "absolute" evenness would result in the same number of substrates utilised upon dilution, given that the inoculum density $\left(>10^{4} \mathrm{cfu} \cdot \mathrm{m}^{-1}\right)$ and incubation time $(72 \mathrm{~h})$ were sufficient. This was substantiated by the dendrogram, which indicated that there was a similarity of more than $99 \%$ amongst the four dilutions (Fig 2b). However, it is unlikely that one would find this sort of evenness in nature. This pattern would rather indicate dominance than "absolute" evenness.

The third artificial community was constructed to reflect an uneven distribution of bacterial species within a community (Table 2). A decline in the number of substrates utilised was observed following serial dilution of the bacterial species mixture (Fig. 3a). This was expected, because some of the species were diluted out and their contribution to substrate utilisation was lost. The undiluted sample (MU0) and the $10^{-1}$ dilution (MU-1) were 91\% similar (Fig. $3 \mathrm{~b})$. This was expected, since a relatively large number of species $\left(>10^{3} \mathrm{cfu} \cdot \mathrm{m}^{-1}\right)$ was present initially in adequate quantities for colour development in the Biolog microtitre plates (Table 2). The $10^{-2}$ (MU-2) dilution was $51 \%$ similar to the undiluted sample (MU0) and $10^{-1}$ dilution (MU-1). This reflected unevenness, as was expected, since the different species in the mixture were not represented in even numbers (Table 2). The $10^{-3}$ (MU-3) dilution was $77 \%$ similar to the $10^{-2}$ (MU-2) dilution, confirming the latter conclusion.

The first natural environment investigated was a hot water spring (HWS) which was representative of an extreme environment. Upon dilution, the percentage substrates utilised, remained consistent for the hot water spring samples (Fig. 4a). This reflected dominance as previously suggested (Fig. 1a). The hot water spring (HWS) data were represented in a dendrogram (Fig. 4b) and all the dilutions were more than $98 \%$ related to one another. Due to the extreme environment (high temperature $\left(>60^{\circ} \mathrm{C}\right)$ ), dominance was expected and indicated. The communities have adapted to these extreme conditions and consist of only a few species, but with large numbers of each species (Connell and Orias, 1964; Kaneko and Atlas, 1977). This supported the hypothesis with regard to dominance.

The second natural environment investigated was activated sludge systems, which was representative of a high species diversity and 
uneven distribution of species (many different species represented by a high number of individuals of each species). Upon dilution, the percentage of substrates utilised from the anaerobic zone of Heidelberg (GN and GP), Vlakplaats (GN and GP) and Hartebeesfontein (GN) water treatment plants decreased (Fig. 5). This indicated a pattern representing unevenness. Tsakane (GN and GP) and Hartebeesfontein (GP) indicated a more even distribution ( $10^{-1}$ to $10^{-3}$ dilutions). The high number of substrates utilised at the lower dilutions $\left(10^{-1}\right.$ and $\left.10^{-2}\right)$ indicated a high initial microbial diversity in the community, but not necessarily evenness of each species. Evenness of each species would be reflected only upon further dilution. It was concluded that the lower the similarity amongst dilutions, the lower the evenness and vice versa.

The $10^{-1}$ and $10^{-2}$ dilutions of the Vlakplaats anaerobic zone inoculated into Biolog GN microtitre plates were 92\% similar, indicating a high initial diversity (Fig. 6). The $10^{-3}$ dilution was $78 \%$ similar to the $10^{-1}$ and $10^{-2}$ dilutions. This indicated a decrease in the species diversity and an uneven distribution of the species. Upon further dilution $\left(10^{-4}\right)$, the similarity decreased to $33 \%$, indicating a further loss of species. This indicated a high initial diversity, but low evenness of species present within this community. The lower the similarity amongst the dilutions, the more uneven the distribution of the species represented.

Figure 7 gives an example of a comparison between GN and GP microplates (containing different substrates), forming one group containing the $10^{-1}$ to $10^{-3}$ dilutions of the Biolog GP microtitre plates with an $83 \%$ similarity. The other group contained the $10^{-1}$ and $10^{-2}$ dilutions of the Biolog GN microtitre plates, with an $87 \%$ similarity. These two groups were $72 \%$ similar.

This indicated a significant difference between the GN and GP profiles. It is important not to compare the GN and GP profiles, since this would show a difference in community structure, which is not related to the true community structure, but rather to the substrates used in the GN and GP plates (Zak et al., 1994). However, considered in isolation (GN or GP), the same conclusions could be drawn for a specific community in terms of functional diversity and evenness. GN and ECO micotitre plates have been compared in a separate study and the results which are not included in this article indicated that these microtitre plates were equally effective in distinguishing microbial metabolic patterns for a number of activated sludge systems. This indicated that a significantly different set of substrates (given that a sufficient number of substrates are incorporated) could be utilised and yet the data generated substantiate the hypothesis that, upon dilution, some members of the community would be lost and that this would be reflected in the metabolic pattern (database) which is generated.

Although the results obtained in this study indicate the usefulness of the Biolog microtitre plate technique for the determination of 98 species diversity, some limitations may make it impossible to draw a valid conclusion. One such scenario would be where the diversity in a system is so high, that irrespective of dilution, all the substrates remain utilised. However, whether this type of community exists, is arguable. Therefore, should a loss of substrate utilisation occur, upon dilution, this technique definitely reflects the extent of evenness in a microbial community, as was illustrated for activated sludge. As proved by this and other studies, the use of substrate utilisation profiles to characterise microbial communities has clear

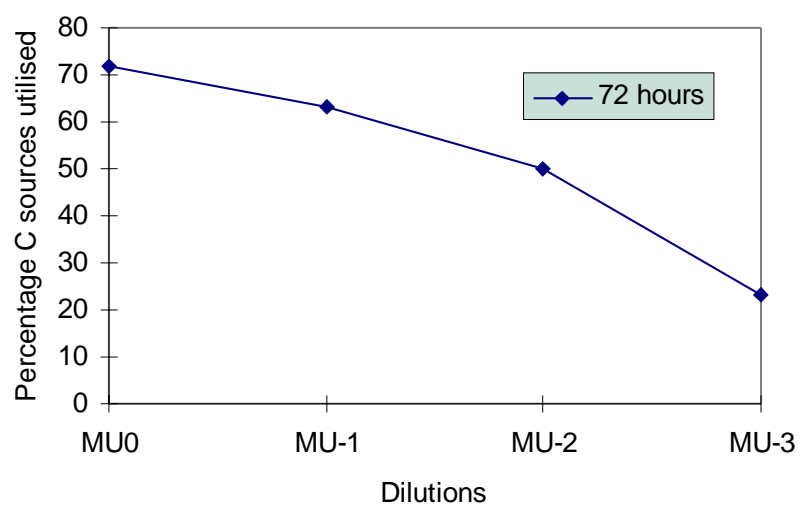

Figure 3(a)

Carbon source utilisation by the mixture of bacterial cultures (MUO, MU-1, MU-2 and MU-3), representative of unevenness (TABLE 2).

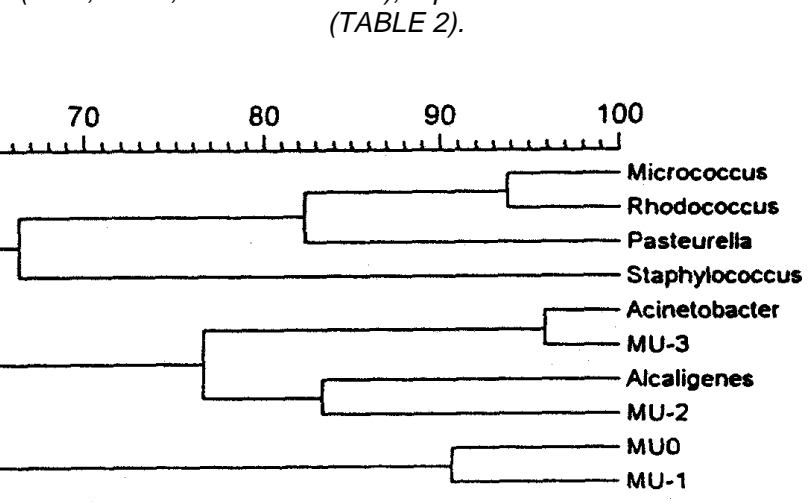

Figure 3(b)

Dendrogram of carbon sources utilised by the mixture of bacterial cultures (MUO, MU-1, MU-2 and MU-3), representative of an uneven distribution of micro-organisms (TABLE 2).

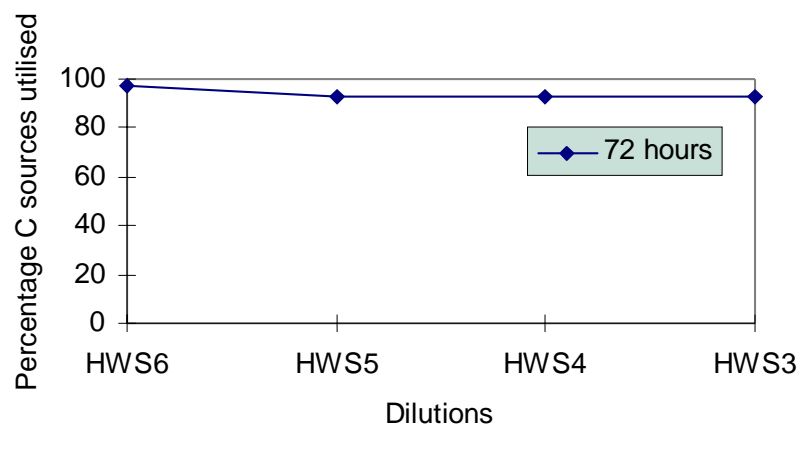

Figure 4(a)

Carbon source utilisation in a Biolog GN microtitre plate by a water sample taken from a hot water spring over a range of

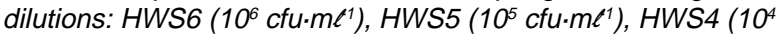
cfu $\left.\cdot m \ell^{1}\right)$ and HWS3 $\left(10^{3} \mathrm{cfu} \cdot \mathrm{m} \ell^{1}\right)$.

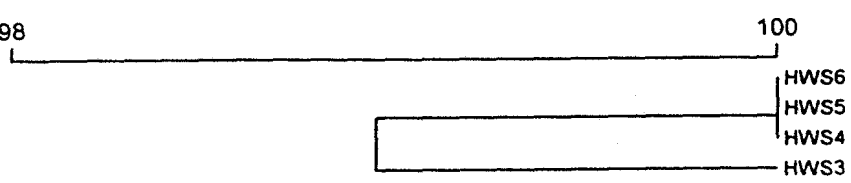

Figure 4(b)

Dendrogram of carbon source utilisation in a Biolog GN microtitre plate by a water sample taken from a hot water spring over a range of dilutions: HWS6 $\left(10^{6} \mathrm{cfu} \cdot \mathrm{m} \ell^{1}\right)$, HWS5 $\left(10^{5} \mathrm{cfu} \cdot \mathrm{m}^{1}\right)$, HWS4 $\left(10^{4} \mathrm{cfu} \cdot \mathrm{m}^{-1}\right)$ and HWS3 $\left(10^{3} \mathrm{cfu} \cdot \mathrm{m} \ell^{-1}\right)$. 


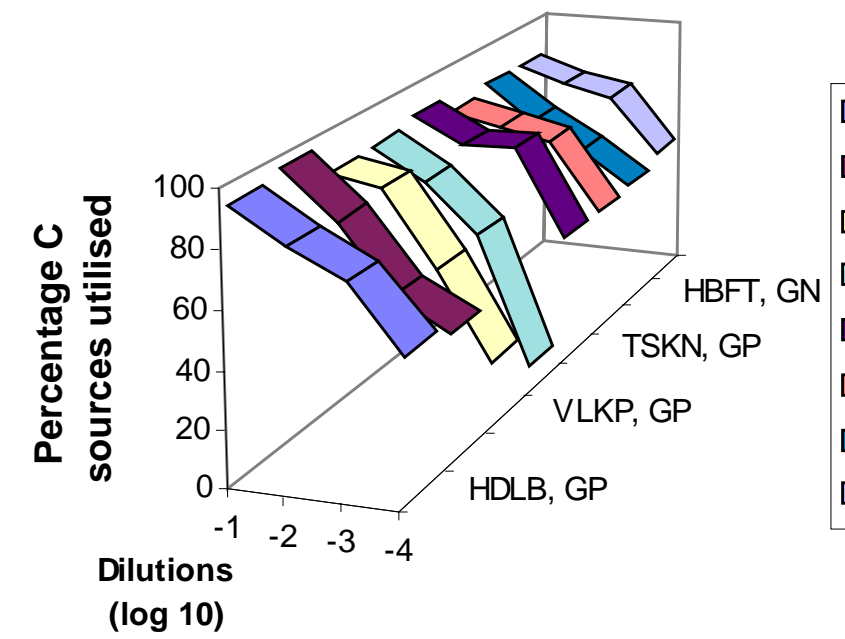

$\square$ HDLB, GP

HDLB, GN

$\square$ VLKP, GP

VLKP, GN

TSKN, GP

TSKN, GN

HBFT, GN

$\square \mathrm{HBFT}, \mathrm{GP}$
Figure 5

Carbon source utilisation in Biolog GN and GP microtitre plates after inoculation with diluted activated sludge samples from the anaerobic zones at the Heidelberg (HDBL), Vlakplaats (VLKP), Tsakane (TSKN) and

Hartebeesfontein (HBFT) water treatment plants
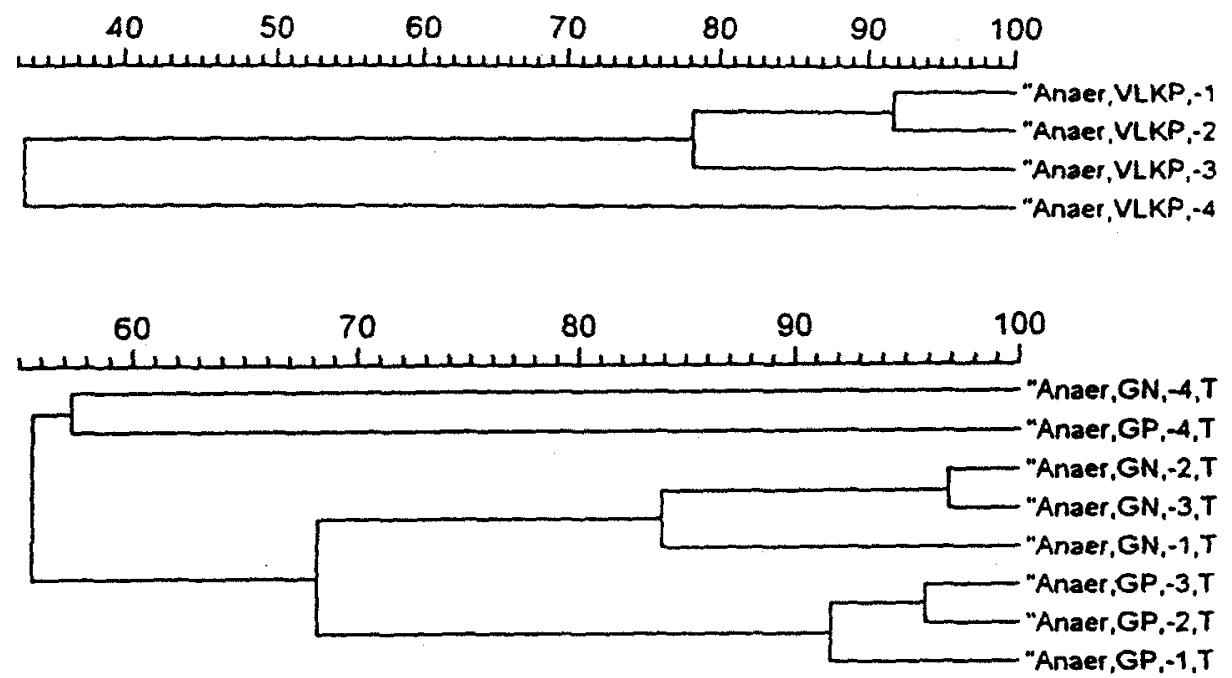

Figure 6

Dendrogram of the Vlakplaats (VLKP) water treatment plant after inoculation of a Biolog GN microtitre plate with activated sludge sampled from the anaerobic (Anaer) zone

Figure 7

Dendrogram of the Tsakane (TSKN) water treatment plant after inoculation of Biolog GN and GP microtitre plates with activated sludge sampled from the anaerobic (Anaer) zone limitations as previously mentioned (Wünsche et al., 1995; Ullrich et al., 1996), but this rapid technique remains a valuable tool for the comparison of microbial communities, provided the data are interpreted cautiously (Smalla et al., 1996).

\section{Conclusions}

- The Biolog system can be used to determine functional (species) diversity and evenness.

- Metabolic profiles in the activated sludge reflected a pattern resembling unevenness, indicated by some of the substrates that are no longer being utilised after the loss of some of the functionality upon dilution.

- The lower the similarity between dilutions, the lower the evenness and vice versa.

- The use of substrate utilisation profiles to characterise microbial communities has clear limitations, but this rapid technique remains a valuable tool for the comparison of microbial communities, provided the data are interpreted cautiously.

\section{Acknowledgements}

We would like to thank the Water Research Commission for their financial support.

\section{References}

AMANN RI, LUDWIG W and SCHLEIFFER K-H (1995) Phylogenetic identification and in situ detection of individual microbial cells without cultivation. Microbiol. Rev. 59 143-159.

BOSSIO DA and SCOW KM (1995) Impact of carbon and flooding on the metabolic diversity of microbial communities in soil. Appl. Environ. Microbiol. 61 4043-4050.

CLOETE TE, BRÖZEL VS and VON HOLY A (1992) Practical aspects of biofouling control in industrial water systems. Int. Biodeterior. Biodegrad. 29 299-341.

CONNELL JH and ORIAS E (1964) The ecological regulation of species diversity. The Am. Nat. 903 399-413.

ELLIS RJ, THOMPSON IP and BAILEY MJ (1995) Metabolic profiling as means of characterizing plant-associated microbial communities. FEMS Microbiol. Ecol. 16 9-18.

ENGELEN B, MEINKEN K, VON WINTZINGERODE F, HEUER H, MALKOMES H-P and BACKHAUS H (1998) Monitoring impact of a pesticide treatment on bacterial soil communities by metabolic and genetic fingerprinting in addition to conventional testing procedures. Appl. Environ. Microbiol. 64 2814-2821.

GARLAND JL and MILLS AL (1991) Classification and characterization of heterotrophic microbial communities on the basis of patterns of community-level sole-carbon-source utilisation. Appl. Environ. Microbiol. 57 2351-2359.

GARLAND JL (1997) Analysis and interpretation of community-level physiological profiles in microbial ecology. FEMS Microbiol. Ecol. 24 289-300. 
GLIMM E, HEUER H, ENGELEN B, SMALLA K and BACKHAUS H (1997) Statistical comparisons of community catabolic profiles. $J$. Microbiol. Methods 30 71-80.

GRIFFITHS BS, RITZ K and WHEATLY RE(1997) Relationship between functional diversity and genetic diversity in complex microbial communities. In: Insam H and Ranger A (ed.) Microbial communities. Functional versus structural approaches. Springer Verlag, Berlin, Heidelberg. New York. 1-9.

GUCKERT JB, CARR GJ, JOHNSON TD, HAMM BG, DAVIDSON DH and KUMAGAI Y (1996) Community analysis by Biolog: Curve integration for statistical analysis of activated sludge microbial habitats. J. Microbiol. Methods 27 183-197.

HAACK SK, GARCHOW H, KLUG MJ and FORNEY LJ (1994) Analysis of factors affecting the accuracy, reproducibility, and interpretation of microbial community carbon source utilisation patterns. Appl. Environ. Microbiol. 61 1458-1468.

HALDEMAN DL and AMY PS (1993) Diversity within a colony morphotype: Implications for ecological research. Appl. Environ. Microbiol. 59 933-935.

HEUER H and SMALLA K (1997) Evaluation of community-level catabolic profiling using Biolog GN microplates to study community changes in potato phyllosphere. J. Microbiol. Methods 30 49-61.

INSAM H, AMOR K, RENNER M and CREPAZ C (1996) Changes in functional abilities of the microbial community during composting of manure. Microb. Ecol. 31 77-87.

KANNEKO T and ATLAS RM (1977) Diversity of bacterial populations in the Beaufort Sea. Nat. 270 596-599.

KERSTERS I, VAN VOOREN L, VERSCHUERE L, VAUTERIN L, WOUTERS A, MERGAERT J, SWINGS J and VERSTRAETE W (1997) Utility of the Biolog system for the characterization of heterotrophic microbial communities. Syst. Appl. Microbiol. 20 439447.

MUYIMA NYO, MOMBA MNB and CLOETE TE (1997) Biological methods for the treatment of wastewaters. In: Cloete TE and Muyima NYO (ed.)Microbial Community Analysis: The Key to the Treatment of Wastewater Treatment Systems. IAWQ, Scientific and Technical Report No 5, University Press, Cambridge. 1-24.
PACE NR, STAHL DA, LANE DL and OLSEN GJ (1986) The analysis of microbial populations by rRNA sequences. Adv. Microbiol. Ecol. 9 $1-55$.

SCHWIEGER F and TEBBE CC (1998) A new approach to utilise PCR single-strand-conformation polymorphism for 16S rRNA gene-based microbial community analysis. Appl. Environ. Microbiol. 64 48704876.

SMALLA K, WACHTENDORF U, KRÖGERRECKLENFORT E and HEUER H (1996) Which members of the bacterial population of the potato phylosphere and rhizosphere contribute most to the BIOLOG community pattern? Abstracts of the Symposium on Bacterial Genetics and Ecology. 93. BAGECO 5, May. Nafplion, Greece.

TORSVIK V, SALTE K, SØRHEIM R and GOKSØYR J (1990) Comparison of phenotypic diversity and DNA heterogeneity in a population of soil bacteria. Appl. Environ. Microbiol. 56 776-781.

ULRICH S, KARRASCH B, HOPPE HG, JESKULKE K and MEHRENS M (1996) Toxic effects on bacterial metabolism of the redox dye 5cyano-2,3-ditolyl tetrazolium chloride. Appl. Environ. Microbiol. 62 4587-4593.

VAN HEERDEN J, EHLERS MM and CLOETE TE (2001) Biolog for the determination of microbial diversity in activated sludge systems. Water Sci. Technol. 43 83-90.

WAGNER M, AMANN R, LEMMER H and SCHLEIFFER K-H (1993) Probing activated sludge with oligonucleotides specific for proteobacteria: Inadequacy of culture-dependent methods for describing microbial community structure. Appl. Environ. Microbiol. 59 15201525.

WINDING A (1994) Fingerprinting bacterial soil communities using Biolog microtitre plates. In: Ritz K, Dighton J and Giller KE (eds.) Beyond the Biomass: Compositional and Functional Analysis of Soil Microbial Communities. Wiley-Sayce, Chichester. 85-94.

WÜNSCHE L, BRÜGGEMANN L and BABEL W (1995) Determination of substrate utilisation patterns of soil microbial communities: An approach to assess population changes after hydrocarbon pollution. FEMS Microbiol. Ecol. 17 295-306.

ZAK JC, WILLIG MR, MOORHEAD DL and WILDMAN HG (1994) Functional diversity of microbial communities: A quantitative approach. Soil Biol. Biochem. 26 1101-1108. 
